\title{
Commentary to "A Case of Recombinant Coxsackievirus A2 Infection with Neurological Complications in Taiwan"
}

'Division of Infectious Diseases, Children's Hospital, China Medical University, school of Medicine, Taichung, Taiwan

Article Info

\section{Article Notes}

Received: November 08, 2017

Accepted: December 27, 2017

\section{${ }^{*}$ Correspondence:}

Dr. Kao-Pin Hwang, Department of Pediatrics, Chief, Pediatric Infectious Disease, and Infection Control, China Medical University Children's Hospital, Number 2, Yu-Der Road, Taichung, 40447, Taiwan;

Email: kapihw@gmail.com

(c) 2017 Hwang KP. This article is distributed under the terms of the Creative Commons Attribution 4.0 International License.
Enteroviruses (EVs) are the predominant pathogens of most childhood illnesses. There are more than one hundred serotypes of EVs belonging to the genus Enterovirus, family Picornaviridae ${ }^{1}$. These viruses were originally classified as polioviruses, coxsackievirus A (CV-As), coxsackievirus B, and echoviruses based on differences in cell tropism, infectivity, antigenicity, and pathogenicity ${ }^{2}$. More recently, these viruses have been re-classified into four species (EVA, EV-B, EV-C, and EV-D) based on their molecular and biological properties ${ }^{3,4}$. The positive-stranded RNA genome of EVs has approximately 7,500 base pairs, and includes three genomic regions (P1, P2, and P3). The P1 region encodes four structural capsid proteins (VP1 to VP4), while the P2 and P3 regions encode seven nonstructural proteins (2A to $2 \mathrm{C}$, and $3 \mathrm{~A}$ to $3 \mathrm{D}$ ). The VP1 capsid protein contains a number of neutralization antigenic sites which are used in virus serotyping ${ }^{5}$. Enterovirus EV-A71 has been reported to cause serious complications of the central nervous system, including encephalitis, meningitis, and poliomyelitis, and there have been several large outbreaks of EV-A71 worldwide in recent decades ${ }^{6-8}$. However, there are limited data on co-circulating nonEV-A71 EV-A strains, which are known to cause outbreaks ${ }^{9,10}$.

Coxsackieviruses are subdivided into groups A and B on the basis of pathogenicity in suckling mice less than 48 hours old (group A caused flaccid paralysis and group B caused spastic paralysis) ${ }^{11}$. Based on past experience, human infections with CV-As are generally mild with the exception of CV-A16. There are a few detailed reports describing the clinical features of coxsackievirus A2 (CV-A2). CV-A2 was previously thought to cause sporadic infections with benign clinical presentation, such as herpangina, and there have been reports of endemic episodes of CV-A2 in Taiwan between 2003 and $2005^{12}$. A CV-A2 epidemic was reported to cause herpangina and hand, foot, and mouth disease (HFMD) among Taiwanese children in 2008 ${ }^{13-14}$, including in two children who had complications of encephalitis and encephalomyelitis, and recovered without any sequelae ${ }^{13}$. Moreover, a natural recombinantCV-A2 was found in Hong Kong in four children with respiratory symptoms, two of whom died during the summer of $2012^{15}$. We previously reported a case of a 9-month-old girl with recombinant CV-A2 infection, who presented with herpangina with encephalomyelitis without long term sequelae ${ }^{16}$. This virus strain was thought to be closely related to the 2012 Hong Kong strain due to the high degree of genetic similarity shared between the two strains. To our knowledge, this was the first case which demonstrated that 
the recombinant $\mathrm{CV}-\mathrm{A} 2$ strain could present with central nervous system involvement.

It was previously reported that in the $2008 \mathrm{CV}-\mathrm{A} 2$ outbreak in Taiwan, most patients with CV-A2 infection presented with herpangina (84-94\%) followed by HFMD, while younger patients mostly presented with respiratory symptoms ${ }^{13,14}$. Febrile seizures were significantly more frequent in patients with CV-A2 infections (8.7\%) compared to the general population ${ }^{14}$. Although uncommon, CVA2 infection may be complicated with encephalitis and encephalomyelitis $(1.1 \%)$ without long term sequelae ${ }^{13}$. Additionally, CV-A2 infection with transverse myelitis has been reported in Korea, 2014 ${ }^{17}$, and in rare cases, patients presented with herpangina with encephalomyelitis. A previous study also reported an outbreak of acute intestinal infection caused by CV-A2, predominantly in Russia in $2010^{18}$. Based on these data, it is important to keep in mind that CV-A2 could be highly transmissible with a large variety of clinical presentations, such as herpangina, HFMD, respiratory or intestinal infections, and even neurological complications.

CV-A2 has been suggested to be the possible pathogen in some lethal cases, including a 15-year-old girl in Germany who had dual infection of CV-A2 and influenza virus B and died in $1969^{19}$. Reverse transcriptase-polymerase chain reaction (RT-PCR) and suckling mouse inoculation were used to detect an enterovirus sequence resembling CVA2 in the stool and spleen of a 10-year-old girl who died suddenly with post-mortem findings of myocarditis ${ }^{20}$. These findings suggested a causal link between CV-A2 and myocarditis. In 2012, Yip et al identified a recombinant CV-A2 in four young children with severe respiratory tract infection, two of whom died ${ }^{15}$. Although it could not be determined whether the virus was the cause of death, the data nevertheless suggested a strong pathogenic role for CV-A2.

Molecular epidemiology studies have demonstrated that circulation of different enteroviruses during outbreaks facilitates recombination of viruses ${ }^{21,22,23,24}$, and this may play an important role in the evolution of enteroviruses. Recombination events have been described previously, especially in the non-capsid (P2 and P3) region of enteroviruses ${ }^{15,25,26}$. In 2011, Hu et al reported that the CV-A2 (A2SD09) and CV-A4 (A4SZ09) strains had serotype-specific homology for the capsid proteins, but shared non-capsid sequences with each other, and the A2SD09 strain clustered with Mongolia strains isolated in $2003^{25}$. Additionally, recombinant CV-A2 strains which caused death in a child in Hong Kong was shown to cluster with the EV-A71 sub-genotype B3 strain SAR/SHA66 in the P2 region, and with the CV-A4 strain SZ/CHN/09 in the P3 region, indicating possible recombination events in these regions. These studies indicated that genetic recombination among CV-A2 strains may be a common evolutionary event. The limited number of complete modern EV-A genome sequences that are available, along with frequent viral recombination events makes it challenging to confirm the origin of these recombinant CVA2 isolates. A comprehensive surveillance for enterovirus A strains, particularly CV-A strains, is needed to expand our understanding of the evolution of these co-circulating viruses.

In conclusion, the case that has been reported as due to CV-A2 infection (E2014041), possibly imported from Hong Kong, caused herpangina and neurological complications without sequelae in Taiwan in $2014^{16}$. This may be the first case which demonstrated that the recombinant CV-A2 strain could complicate with central nervous system disorders. Genetic findings suggested that this could be a particularly aggressive and neurotropic strain of CV-A2. CV-A2 is the predominant strain causing several endemic and epidemic outbreaks in Taiwan, and circulation of these viruses during outbreaks may facilitate viral recombination and the emergence of a virus causing a new disease manifestations. It is therefore important to institute a continued surveillance of CV-A2 circulation, based on modern genome sequencing, in order to monitor the molecular epidemiology of such outbreaks, and to track emerging recombination events.

\section{References}

1. Oberste MS, Maher K, Nix WA, et al. Molecular identification of 13 new enterovirus types, EV79-88, EV97, and EV100-101, members of the species Human Enterovirus B. Virus Res. 2007; 128(1-2): 34-42.

2. Melnick JL. Fields virology. 3rd ed. Philadelphia, PA.: LippincottRaven; 1996.

3. Hyypiä T, Hovi T, Knowles NJ, et al. Classification of enteroviruses based on molecular and biological properties. J Gen Virol. 1997; 78(1): 1-11.

4. Oberste MS, Maher K, Michele SM, et al. Enteroviruses 76, 89, 90 and 91 represent a novel group within the species Human enterovirus A. J Gen Virol. 2005; 86(2): 445-51.

5. Nix WA, Oberste MS, Pallansch MA. Sensitive, seminested PCR amplification of VP1 sequences for direct identification of all enterovirus serotypes from original clinical specimens. J Clin Microbiol. 2006; 44(8): 2698-704.

6. Nagy G, Takátsy S, Kukán E, et al. Virological diagnosis of enterovirus type 71 infections: experiences gained during an epidemic of acute CNS diseases in Hungary in 1978. Arch Virol. 1982; 71(3): 217-27.

7. Chan LG, Parashar UD, Lye MS, et al. Deaths of children during an outbreak of hand, foot, and mouth disease in sarawak, malaysia: clinical and pathological characteristics of the disease. For the Outbreak Study Group. Clin Infect Dis. 2000; 31(3): 678-83.

8. Ho M, Chen ER, Hsu KH, et al. An epidemic of enterovirus 71 infection in Taiwan. Taiwan Enterovirus Epidemic Working Group. N Engl J Med. 1999; 341(13): 929-35.

9. Blomqvist $\mathrm{S}$, Klemola $\mathrm{P}$, Kaijalainen $\mathrm{S}$, et al. Co-circulation of coxsackieviruses A6 and A10 in hand, foot and mouth disease outbreak in Finland. J Clin Virol. 2010; 48(1): 49-54.

10. Lu QB, Zhang XA, Wo Y, et al. Circulation of Coxsackievirus A10 and 
A6 in hand-foot-mouth disease in China, 2009-2011. PLoS One. 2012; $7(12)$ : e52073

11. Hyypiä T, Kallajoki M, Maaronen M, et al. Pathogenetic differences between coxsackie A and B virus infections in newborn mice. Virus Res. 1993 Jan; 27(1): 71-8.

12. Tseng FC, Huang $\mathrm{HC}$, Chi $\mathrm{CY}$, et al. Epidemiological survey of enterovirus infections occurring in Taiwan between 2000 and 2005: analysis of sentinel physician surveillance data. J Med Virol. 2007; 79(12): 1850-60.

13. Chen SP, Huang YC, Li WC, et al. Comparison of clinical features between coxsackievirus $\mathrm{A} 2$ and enterovirus 71 during the enterovirus outbreak in Taiwan, 2008: a children's hospital experience. J Microbiol Immunol Infect. 2010; 43(2): 99-104.

14. Lee MH, Huang LM, Wong WW, et al. Molecular diagnosis and clinical presentations of enteroviral infections in Taipei during the 2008 epidemic. J Microbiol Immunol Infect. 2011; 44(3): 178-83.

15. Yip CC, Lau SK, Woo PC, et al. Recombinant coxsackievirus A2 and deaths of children, Hong Kong, 2012. Emerg Infect Dis. 2013 Aug; 19(8): 1285-8.

16. Yen TY, Huang YP, Hsu YL, et al. A case of recombinant coxsackievirus A2 infection with neurological complications in Taiwan. J Microbiol Immunol Infect. 2016 Dec; 18(16)30148-7. doi: 10.1016/j. jmii.2016.08.012.

17. Kim H, Kang B, Hwang S, et al. Clinical and enterovirus findings associated with acute flaccid paralysis in the Republic of Korea during the recent decade. J Med Virol. 2014; 86(9): 1584-9.

18. Demina AV, Ternovoř VA, Darizhapov BB, et al. Outbreak of acute enterovirus intestinal infection in Sakhalin region in August 2010. Vestn Ross Akad Med Nauk. 2012; (2): 64-8.

19. Adamczyk B, Klier G, Deicke P. Lethal double infection with coxsackie virus A2 and influenza virus B in a 15-year-old girl. Z Gesamte Inn Med. 1969; 24(24): 917-21.

20. Bendig JW, O’Brien PS, Muir P, et al. Enterovirus sequences resembling coxsackievirus A2 detected in stool and spleen from a girl with fatal myocarditis. J Med Virol. 2001; 64(4): 482-6.

21. Huang SC, Hsu YW, Wang HC, et al. Appearance of intratypic recombination of enterovirus 71 in Taiwan from 2002 to 2005. Virus Res. 2008; 131(2): 250-9.

22. Jegouic S, Joffret ML, Blanchard C, et al. Recombination between polioviruses and co-circulating Coxsackie A viruses: role in the emergence of pathogenic vaccine-derived polioviruses. PLoS Pathog. 2009 May; 5 (5): e1000412.

23. Oprisan G, Combiescu M, Guillot S, et al. Natural genetic recombination between co-circulating heterotypic enteroviruses. J Gen Virol. 2002; 83(9): 2193-200.

24. Zhang Y, Zhu Z, Yang W, et al. An emerging recombinant human enterovirus 71 responsible for the 2008 outbreak of hand foot and mouth disease in Fuyang city of China. Virol J. 2010; 7: 94.

25. Hu YF, Yang F, Du J, et al. Complete genome analysis of coxsackievirus A2, A4, A5, and A10 strains isolated from hand, foot, and mouth disease patients in China revealing frequent recombination of human enterovirus A. J Clin Microbiol. 2011; 49(7): 2426-34.

26. Huang YP, Lin TL, Hsu LC, etal. Genetic diversity and C2-like subgenogroup strains of enterovirus 71, Taiwan, 2008. Virol J. 2010; 7: 277. 